\title{
RECURSOS HUMANOS DE LAS REGIONES ESPAÑOLAS
}

\section{Jacinto Rodríguez Osuna}

\section{INTRODUCCION}

Los estudios regionales son tema de constante actualidad justificados, en gran parte, por los desequilibrios que se han ido produciendo entre las regiones, en los países de economía de mercado, durante el proceso de crecimiento económico.

Como es conocido, «cuando en una economía las fuerzas de mercado actúan con cierta libertad se produce una concentración espacial (y de otros aspectos), que quizá desde un punto de vista estrictamente económico puede admitirse para lograr una máxima productividad de la actividad económica en las primeras etapas del crecimiento ${ }^{1}$. La existencia de una mejor infraestructura, de factores de producción abundantes y de mejor calidad, la tradición empresarial, la existencia de mercados cercanos, etc., constituyen un conjunto de circunstancias que favorecen la expansión en torno a determinadas áreas. Como consecuencia de ello, la distancia entre las regiones se va haciendo mayor, produciéndose un trasvase de recursos humanos y financieros desde las áreas menos desarrolladas a las más desarrolladas, con

- Martinez Cortiña, Rafael, y otros: Regionalización de la economía española, Fondo para la investigación económica y social de la Confederación de Cajas de Ahorro, Madrid, 1975, p. 14. 
lo que la distancia se va agrandando y las diferencias se van haciendo cada vez más notorias.

Este esquema elemental recoge, de forma simplificada, la trayectoria que han seguido muchos países en su desarrollo económico, y que ha dado lugar a la saturación industrial y deseconomías externas en determinadas áreas, y al estancamiento y la pobreza y, en definitiva, a situaciones más difícil. mente reversibles, en otras.

Centrándonos en el caso de España no resulta complejo enmarcar la trayectoria de nuestra economía en estas coordenadas. En la primera mitad del presente siglo el desarrollo económico se polarizó en pocas áreas, que crecieron a un ritmo superior a las demás, pero no con suficiente fuerza como para movilizar, en gran escala, los recursos humanos y financieros del resto del país. Por eso hasta 1950 la media anual de migraciones interiores se mantiene casi constante y dentro de unos límites muy bajos en comparación a lo que ocurriría más tarde ${ }^{2} \mathrm{y}$, además, los crecimientos intercensales de población, a nivel de provincia, son siempre positivos, salvo rarísimas excepciones. A partir de la década de los cincuenta el proceso señalado anteriormente se desencadena y sus manifestaciones son múltiples. La economía crece muy deprisa y en torno a determinadas áreas, la producción se concentra, las migraciones interiores se multiplican, la mayor parte de las provincias españolas muestran saldos migratorios negativos y 18 terminan la década de los cincuenta con crecimientos intercensales negativos. Era el comienzo de un fuerte proceso de concentración de la producción y de la población en determinadas áreas, y del anquilosamiento y desertización en otras.

La situación creada era la más propicia para que se despertara la preocupación por los estudios regionales y así, a partir de entonces, se empiezan a multiplicar los estudios en esta dirección. Román Perpiñá Gráu ${ }^{3}$, Luis del Hoyo ${ }^{4}$, Juan Plaza Prieto ${ }^{5}$, José Luis Sampedro ${ }^{6}$, José González Paz ${ }^{7}$, Ramón Tamames ${ }^{8}$, Rafael Martínez Cortiña ${ }^{9}$, son algunos de los múltiples

2 Según Alfonso García Barbancho: Las migraciones interiores españolas, Instituto de Desarrollo Económico, Madrid, 1970, la media anual de migraciones de 1900 a 1950 osciló entre 86.000 y 105.000 . Años más tarde, en torno a los setenta, la media anual de migraciones interiores se situaría cerca de los $550.000 \mathrm{mi}$ grantes.

${ }^{3}$ Román Perpiña Grad: De estructura económica y economía hispana, Rialp, Madrid, 1952.

4 Luis del Hoyo SaINz: Antropodemografía española. Regiones y razas, Real Sociedad Geográfica, Madrid, 1942.

s Juan Plaza Prieto: Regiones económicas homogéneas españolas, Centro de Estudios Económicos, Información y síntesis del INI, Madrid, 1964.

- José Luis Sampedro y otros: Perfiles económicos de las regiones españolas, Sociedad de Estudios y Publicaciones del Banco Urquijo, Madrid, 1964.

7 José González Paz: Regiones socioeconómicas españolas, Centro de Estudios

Económicos, Información y sintesis del INI, Madrid, 1964.

- Ramón Tamames: Estructura económica de España, Guadiana, 1974.

9 Rafael Martínez Cortiña y otros: Regionalización..., op. cit. 
autores que se han enfrentado con el tema regional español. Si de alguna forma hubiera que señalar objetivos comunes de estos estudios habría que resaltar que se han preocupado de:

- la división regional;

- el estudio de los desequilibrios espaciales;

- la política regional.

Muchos de los autores que han estudiado los desequilibrios regionales españoles parten de las llamadas regiones históricas, pero otros, como los aquí señalados, empiezan por seleccionar una serie de criterios, y en base a ellos, hacen su propia división regional. Los resultados de estas investigaciones son diversos y no siempre coincidentes, pero constituyen puntos de partida valiosos, tanto para el análisis de los desequilibrios regionales como para fijar áreas perfectamente acotadas que puedan servir de base para el relanzamiento de la economía regional con la finalidad de desarrollar un crecimiento armónico a nivel nacional en que se sustituya una planificación sectorial-centralista por una planificación sectorial-regional.

Teniendo como marco de referencia este contexto el estudio que aquí se plantea tiene como objetivo analizar los recursos humanos de las regiones españolas, lo cual lleva consigo incluir en nuestro discurso el estudio de los desequilibrios regionales, desde el punto de vista de la población.

La preocupación por hacer una división regional pierde interés en este trabajo porque esa división regional se va construyendo desde otras esferas, las políticas. Cualquiera que sea la validez de esa regionalización, en parte coincidente con la histórica, el hecho es que el proceso político español va dando lugar a las autonomías que van a servir de base para esa política regional a que se viene aludiendo. En nuestro trabajo se parte, por tanto, de la división regional autonómica ${ }^{10}$ y en base a ella se van a estudiar los recursos humanos con la metodología que se irá explicando a lo largo del análisis. De esta manera se pretende ofrecer una visión global del punto de partida de cada una de las regiones, en comparación con las otras, en su nueva andadura autonómica. Hay que señalar en seguida que las regiones no son homogéneas, y que en muchas existen diferencias importantes entre las provincias que las componen. En un estudio como el presente se va a perder la riqueza que supondría un análisis provincial, pero, sin embargo, se va a conseguir una aproximación global a los desequilibrios de que se viene hablando, y además, en algunos casos, se hará alusión a las provincias para completar el análisis.

Nuestro estudio se plantea desde el análisis de variables demográficas,

10 En alguna parte del territorio nacional no están definidas de forma definitiva las regiones autonómicas. Aunque pueda haber algunas modificaciones, éstas harán variar muy poco la división regional que aquí se ofrece. 
respondiendo al enfoque monográfico de este número de la revista. Ello no implica que lo que se va a hacer sea un estudio puramente demográfico. Las variables demográficas que aquí se utilizan permiten captar la diferencia de recursos humanos de que se parten cada una de las regiones y la dinámica de esos recursos, pero, además, y de forma global, el desarrollo económico diferente de cada región, como se verá más adelante.

El trabajo se va a dividir en dos grandes apartados. En el primero se hará referencia a la población y su dinámica, y en el segundo se abordará la relación entre demografía y economía, partiendo del análisis del caso español y referida a las variables utilizadas en este trabajo.

Adelantándonos al desarrollo del trabajo hay que señalar, desde ahora, que el estudio no va a ser solamente estático, por la sencilla razón de que las últimas décadas han sido las de mayor dinamismo en la evolución de la economía y ello se refleja de forma inexorable en el comportamiento de las variables demográficas. De ahí que sea imprescindible ir viendo los cambios que se producen, en qué regiones se producen y por qué se producen.

\section{LA POBLACION}

\section{Distribución espacial de la población}

Una primera aproximación al estudio de los recursos humanos de cada región podría consistir en el análisis de la distribución espacial de la población tomando como unidades territoriales las regiones autonómicas a que nos venimos refiriendo. En este sentido las regiones con mayor volumen de población, en 1975, serían: Andalucía, 17 por 100; Cataluña, 16 por 100; Madrid, 12 por 100, Valencia, 9 por 100; Galicia, 7 por 100, y CastillaLeón, 7 por 100 , ya que su población conjunta representa el 68 por 100 del total nacional. Sin embargo, esta manera de proceder no tiene en cuenta ni la dinámica de esas regiones ni la relación hombre/espacio, temas de especial importancia en el análisis de la distribución espacial de la población.

A la dinámica de la distribución espacial se ha hecho alusión en la introducción, señalando cómo la década de los cincuenta marca una frontera entre dos etapas bien definidas separadas por un proceso económico, el comienzo del llamado desarrollo español.

El cambio de ritmo entre una y otra etapa en la distribución espacial de la población se detecta de múltiples maneras: aumento considerable de las migraciones interiores, la aparición de provincias con crecimientos intercensales negativos, los ritmos cada vez más distantes de crecimiento poblacional de cada provincia. Este último hecho se constata fácilmente. Los 
crecimientos intercensales provinciales extremos se alejan cada vez más, como puede verse en los datos siguientes:

\begin{tabular}{|c|c|c|c|}
\hline Décadas & $\begin{array}{l}\text { Provincia que } \\
\text { más decrece }\end{array}$ & $\begin{array}{l}\text { Provincia que } \\
\text { más crece }\end{array}$ & Diferencia \\
\hline $\begin{array}{lllllllll}1941-1950 & \ldots & \ldots & \ldots & \ldots & \ldots & \ldots & \ldots & \ldots \\
1951-1960 & \ldots & \ldots & \ldots & \ldots & \ldots & \ldots & \ldots & \ldots \\
1961-1970 & \ldots & \ldots & \ldots & \ldots & \ldots & \ldots & \ldots & \ldots\end{array}$ & $\begin{array}{r}1,18 \\
-\quad 9,71 \\
-21,83\end{array}$ & $\begin{array}{l}21,93 \\
35,30 \\
47,06\end{array}$ & $\begin{array}{l}23,11 \\
45,01 \\
68,89\end{array}$ \\
\hline
\end{tabular}

La distancia de 23 puntos, en la década de los 40, se eleva a 69 en la década de los 60, debido al aumento de la diferencia de crecimiento entre unas y otras provincias. A un resultado en la misma dirección se llega por otro camino: hallando la media de los crecimientos intercensales provinciales, para cada década, y comparando su dispersión a través del coeficiente de variación.

En este caso se obtienen estos resultados:

\begin{tabular}{|c|c|c|}
\hline & Décadas & $\begin{array}{l}\text { Coeficiente } \\
\text { de variación }\end{array}$ \\
\hline $\begin{array}{l}1941-1950 \\
1951-1960 \\
1961-1970\end{array}$ & $\begin{array}{ccccccc}\ldots & \ldots & \ldots & \ldots & \ldots & \ldots & \ldots \\
\ldots & \ldots & \ldots & \ldots & \ldots & \ldots & \ldots \\
\ldots & \ldots & \ldots & \ldots & \ldots & \ldots & \ldots \\
\ldots & \ldots & \ldots & \ldots & \ldots & \ldots & \ldots\end{array}$ & $\begin{array}{l}0,76 \\
2,15 \\
4,52\end{array}$ \\
\hline
\end{tabular}

Hay, por tanto, un cambio de ritmo en la dinámica de las provincias, que se va a reflejar en la evolución de la relación hombre/espacio. Analizando ambos hechos nos podemos aproximar mejor a nuestro intento de comprender la distribución de la población en el territorio nacional.

\section{El ritmo de crecimiento}

En el cuadro 1 se refleja el crecimiento de la población en cada uno de los períodos señalados, así como la relación hombre/espacio, densidad, en tres momentos distintos.

Analizando primero el crecimiento intercensal se observa que las cuatro primeras regiones, Madrid, País Vasco, Cataluña y Canarias, son las de máximo crecimiento en uno y otro período, situándose muy por encima del crecimiento nacional. La evolución del resto de las regiones es dispar y conviene analizarla por separado en las dos etapas.

De 1900 a 1950 todas las regiones tienen crecimiento positivo y la distancia entre Andalucía y Logroño, la de máximo y mínimo crecimiento, 
exceptuadas las cuatro primeras, es sólo de 37 puntos en el transcurso de los cincuenta años. Por otra parte, las diferencias de crecimiento no están en función de la España interior y la periférica ", sino más bien de la España que se sitúa al sur de Madrid y la que se sitúa al norte. Así resulta que Andalucía, Extremadura y la Mancha tienen un crecimiento muy superior al de Castilla-León, Galicia y Logroño.

\section{CUADRO 1}

Crecimiento de la población. Densidades en relación a la media nacional

\begin{tabular}{|c|c|c|c|c|c|c|c|}
\hline \multicolumn{3}{|l|}{ Regiones } & \multicolumn{2}{|c|}{$\begin{array}{l}\text { Crecimiento } \\
\text { de la población }\end{array}$} & \multicolumn{3}{|c|}{$\begin{array}{l}\text { Densidades en relación a la } \\
\text { media nacional }\end{array}$} \\
\hline & & & $1900-50$ & $1951-75$ & 1900 & 1950 & 1.975 \\
\hline Madrid $\ldots \ldots \ldots$. & $\ldots$ & $\ldots$ & 149 & 122 & 263 & 434 & 774 \\
\hline País Vasco .... & $\ldots$ & & 76 & 95 & 236 & 276 & 395 \\
\hline Cataluña ......... & $\ldots$ & $\ldots$ & 67 & 75 & 167 & 182 & 248 \\
\hline $\begin{array}{llll}\text { Canarias } & \ldots & \ldots & \ldots\end{array}$ & $\ldots$ & $\ldots$ & 121 & 71 & 134 & 196 & 247 \\
\hline $\begin{array}{lllll}\text { Valencia } & \ldots & \ldots & \ldots\end{array}$ & $\ldots$ & $\ldots$ & 45 & 48 & 185 & 179 & 203 \\
\hline Baleares $\ldots \ldots \ldots$ & $\ldots$ & $\ldots$ & 35 & 50 & 169 & 152 & 177 \\
\hline $\begin{array}{lllll}\text { Navarra } & \ldots & \ldots & \ldots\end{array}$ & $\ldots$ & $\ldots$ & 24 & 26 & 80 & 66 & 65 \\
\hline $\begin{array}{llll}\text { Asturias } & \ldots & \ldots & . .\end{array}$ & $\ldots$ & $\ldots$ & 42 & 23 & 161 & 152 & 146 \\
\hline Santander . ...... & $\ldots$ & $\ldots$ & 47 & 21 & 141 & 138 & 130 \\
\hline Murcia.$\ldots \ldots$. & $\ldots$ & $\ldots$ & 31 & 17 & 138 & 121 & 109 \\
\hline Galicia $\ldots \ldots \ldots$ & $\ldots$ & $\ldots$ & 31 & 4 & 182 & 162 & 127 \\
\hline Andalucía . ... .. & $\ldots$ & $\ldots$ & 58 & 9 & 110 & 115 & 98 \\
\hline $\begin{array}{lllll}\text { Logroño } & \ldots & \ldots & \ldots\end{array}$ & $\ldots$ & $\ldots$ & 21 & 5 & 102 & 82 & 67 \\
\hline Aragón & $\ldots$ & $\ldots$ & 23 & 5 & 51 & 41 & 34 \\
\hline Castilla-León . . & $\ldots$ & $\ldots$ & 24 & -11 & 66 & 55 & 38 \\
\hline La Mancha ... .. & $\ldots$ & $\ldots$ & 46 & -19 & 47 & 46 & 29 \\
\hline Extremadura & $\ldots$ & $\ldots$ & 55 & --22 & 57 & 59 & 36 \\
\hline TOTAL ... & $\ldots$ & $\ldots$ & 51 & 28 & $100=36,8$ & $100=55,4$ & $100=71,3$ \\
\hline
\end{tabular}

Fuente: Explotación de datos censales y padrones.

En el período siguiente, el crecimiento poblacional de las regiones sigue un ritmo muy diferente. En primer lugar, las cuatro primeras regiones crecen a un ritmo medio anual mucho más elevado. Las diferencias de crecimiento en el resto de las regiones son ahora mucho más acusadas, a pesar de ser un período de sólo veinticinco años. Entre Valencia y Extremadura se da una diferencia de 70 puntos, casi el doble del que se daba en las regiones extremas del período anterior.

"Sobre la distribución de la población en las provincias interiores y las marítimas, existen varios estudios a los que se aludirá más adelante. En general, y con las matizaciones que se señalarán. la población tiende a situarse en las provincias periféricas. 
A la vista de los crecimientos poblacionales, desde 1950 (véase cuadro 1), se podrían establecer cuatro grupos de regiones de dinámica bien distinta:

- Las cuatro primeras del cuadro: Madrid, País Vasco, Cataluña y Canarias, tenían un crecimiento elevado desde principios de siglo, aumentando su ritmo a partir de 1950. A estas regiones se unen Valencia y Baleares en la segunda etapa considerada. En conjunto, constituyen tres grandes áreas, que se han ido consolidando poco a poco, y que tienen la más alta densidad nacional. Estas tres grandes áreas son la de Madrid, la del País Vasco, que con un origen en Guipúzcoa y Vizcaya extendió su dinamismo a Alava en 1950, y la formada por Cataluña, Valencia y Baleares, regiones con muchas similitudes culturales y cuyo desarrollo se originó en torno a Barcelona y Valencia, para extenderse, también a partir de 1950 , al resto de las provincias.

- Navarra, Asturias, Santander y Murcia, que se sitúan con crecimientos inferiores a la media nacional, pero no muy distante de la misma. Su densidad, elevada en 1900, salvo en Navarra, desciende en términos relativos desde principio de siglo.

- Galicia, Andalucía, Logroño y Aragón, cuyo crecimiento medio anual se sitúa muy próximo a cero, con densidades superiores a la nacional a principio de siglo, salvo en Aragón, pero en descenso rápido en términos comparativos.

- Castilla-León, Mancha y Extremadura, con crecimientos negativos elevados y con una densidad muy baja.

Aun partiendo de datos tan agregados como los regionales, se pone en evidencia que se da una clara diferencia de ritmo de crecimiento entre la España interior y la periférica, sobre todo a partir de 1950. Si se quisiera llegar a un análisis más detallado habría que recurrir a datos provinciales, que evidencian cómo a partir de 1950 prácticamente toda la periferia tiene crecimiento poblacional, más o menos intenso, pero positivo, y todo el interior, salvo raras excepciones, crecimiento negativo. El tema ha sido desarrollado en otro trabajo anterior ${ }^{12} y$ de él se volverá a hablar en el epígrafe siguiente con mayor detalle.

Hay una periferia muy dinámica, la de las provincias vascas ${ }^{13}$ catalanas y valencianas, y otra mucho menos dinámica, la que se refiere al resto de las provincias peninsulares marítimas. Como también hay una España interior dispar, aunque aquí las diferencias sean mucho menores. Madrid des-

12 Jacinto Rodricuez Osuna: "Distribución espacial de la población y desarrollo económico en España", en REIS, octubre-diciembre 1978.

13 Los datos que se analizan llegan hasta 1975 y no tienen en cuenta, por tanto, la evolución posterior. 
taca como un islote muy dinámico poblacionalmente y lo mismo ocurre con Valladolid y Zaragoza, aunque estas últimas provincias sólo desde época reciente $y$ con un dinamismo mucho menor.

En resumen, se podría concluir que, sobre todo a partir de 1950 , se han configurado áreas de dinamismo bien diferente en el territorio nacional:

- La España marítima del Nordeste, Este y las Islas (País Vasco, Cataluña, Valencia y las Islas), que, en conjunto, es la de máximo dinamismo demográfico.

- El resto de España marítima, de escaso dinamismo, pero con crecimientos poblacionales positivos.

- La España interior dinámica, constituida por la provincia de Madrid, Valladolid y Zaragoza. La primera de máximo dinamismo y las otras dos con una clara progresión que destaca dentro del entorno que las rodea.

- La España interior, que se desertiza y que viene constituida por todas las provincias interiores, excepto las señaladas.

\section{La relación hombre/espacio en la España interior y la periférica}

El análisis anterior, en que se detecta la clara tendencia de la población a trasladarse hacia la periferia, sobre todo a partir de 1950 , puede completarse con nuevos estudios, partiendo de los trabajos de Perpiñá Gráu ${ }^{14}$, del Foessa $70^{15}$, de Rodríguez Osuna ${ }^{16}$.

Perpiñá Gráu, a través de una investigación continuada en esta dirección, insistirá en que la población sigue el curso de la riqueza y ésta se halla en Madrid y, en la periferia, en torno sobre todo a seis grandes áreas, Barcelona, Valencia, Cádiz-Sevilla, Lisboa-Oporto, La Coruña-Pontevedra y País Vasco. Si bien este esquema podía ser válido a principio de siglo, en la actualidad habría que completarlo con nuevas matizaciones. El ritmo de crecimiento de Barcelona, Madrid, País Vasco y Valencia es muy superior al resto de las provincias señaladas por el autor, pero además el crecimiento de Barcelona, País Vasco y Valencia se va transmitiendo a las provincias limítrofes, hecho que no ocurre en el resto de las provincias señaladas. Para el Foessa 70 el crecimiento de la periferia no abarca a todas las provincias de este área, sino a una determinada franja a lo largo de la costa que muy bien pudiera identificarse con los partidos judiciales con acceso al mar.

Rodríguez Osuna clasifica las provincias en función de su crecimento y, manteniendo la tesis del mayor crecimiento de la periferia, concreta más el crecimiento diferencial de unas y otras provincias.

14 Román Perpiñ Grau: Corología: teoria estructural y estructurante de la población de España 1900-1950, CSIC, Madrid, 1954.

${ }^{13}$ Fundación FOESSA 70: Informe sociológico sobre la situación social de España 1970, Euramérica, Madrid, 1970.

is Véase nota 12. 
Otra manera de plantearse el problema es a través del análisis de la evolución de los saldos migratorios (véase cuadro 2 ).

\section{CUADRO 2}

Clasificación de las provincias según el signo de los saldos migratorios

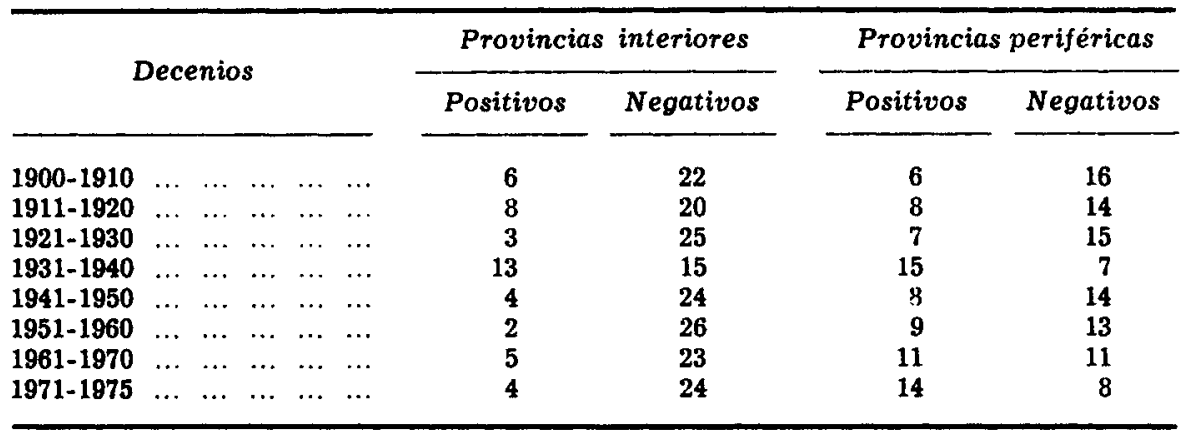

Fuentr: Explotación de datos tomados del III Plan de Desarrollo.

De las veintiocho provincias interiores españolas la mayoría tienen saldos migratorios negativos a lo largo de todo el siglo, salvo en el decenio 19311940, anómalo por la existencia de la guerra civil española. De las veintidós provincias periféricas, también una parte tiene saldos migratorios negativos, pero el número de provincias afectada es cada vez menor, de tal forma que en el último quinquenio, las provincias con saldos migratorios positivos son muchas más que las provincias con saldos migratorios negativos. A partir de 1960 se da un cambio en el proceso que obedece a todo lo que se ha constatado tantas veces: el proceso de redistribución de la población se acelera a favor de determinadas provincias, que son fundamentalmente las periféricas.

Todo este análisis desemboca en la distinta relación hombre/espacio en la España interior y en la periférica, que si bien se consolida en los últimos años, tiene sus raíces en muchos años atrás.

De las veintiocho provincias interiores solamente cuatro tienen una densidad superior a la nacional, en 1900, para quedar reducidas a tres en 1975 (cuadro 3). De las provincias periféricas, sólo una tiene densidad inferior a la nacional en 1900 y cinco al final del período analizado, es decir, en 1975.

Comparando uno y otro cuadro, el 2 y el 3, se concluye fácilmente que son precisamente las provincias interiores las de mayores saldos migratorios negativos y las de menor densidad, mientras que las provincias periféricas tienen saldos migratorios positivos y una densidad mucho más elevada. 
Profundizando un poco más en el análisis de densidades, que creemos fundamental (véanse cuadros $+y 5$ ), se puede llegar a conclusiones más matizadas.

\section{CUADRO 3}

Provincias con densidades superiores o inferiores a la del resto de las provincias de su área

\begin{tabular}{|c|c|c|c|}
\hline & \multicolumn{3}{|c|}{$A \bar{n} \circ \mathrm{s}$} \\
\hline & 1900 & 1950 & 1975 \\
\hline $\begin{array}{l}\text { Provincias interiores con den- } \\
\text { sidad superior a la nacional }\end{array}$ & $\begin{array}{l}\text { Logrono } \\
\text { Sevilla } \\
\text { Orense } \\
\text { Madrid }\end{array}$ & $\begin{array}{l}\text { Córdoba } \\
\text { Jaén } \\
\text { Orense } \\
\text { Madrid }\end{array}$ & $\begin{array}{l}\text { Alava } \\
\text { Sevilla } \\
\text { Madrid }\end{array}$ \\
\hline $\begin{array}{l}\text { Provincias periféricas con den- } \\
\text { sidad inferior a la nacional }\end{array}$ & Huelva & $\begin{array}{l}\text { Almería } \\
\text { Huelva } \\
\text { Castellón } \\
\text { Lugo }\end{array}$ & $\begin{array}{l}\text { Huelva } \\
\text { Almeria } \\
\text { Granada } \\
\text { Lugo } \\
\text { Castellón }\end{array}$ \\
\hline
\end{tabular}

El cuadro 4 hace referencia a las provincias con densidad inferior a la nacional, reflejándose en el mismo la posición relativa de cada una de ellas. De las veintiocho provincias interiores, veinticuatro aparecen en el cuadro, y de las periféricas, solamente una. Ello significa que la densidad de las provincias interiores es muy baja y, a la vez, muy distante de la de las provincias periféricas.

El cuadro refleja, por otra parte, la evolución de las densidades a lo largo de los dos períodos más significativos, 1900-1950 y 1950-1975. En el primer período, tres provincias, Ciudad Real, Córdoba y Jaén, ganan densidad en términos relativos, mientras que en el segundo, sólo Alava gana densidad y dieciséis la pierden, situándose estas dieciséis provincias en todas las regiones interiores, confirmándose que el proceso de desertización afecta a la España interior, a la España de bajas densidades.

Las conclusiones a que se llega, por este camino, completan las ya apuntadas en páginas anteriores:

- Las densidades de las provincias interiores son muy inferiores a las de las provincias periféricas.

- La mayor pérdida de dinamismo se da en las provincias interiores, las de menor densidad.

- El período de mayor desertización de la España interior hay que situarlo a partir de 1950 . 


\section{CUADRO 4}

Provincias que en 1900 tenian densidad inferior a la media nacional. Evolución de densidades

\begin{tabular}{|c|c|c|c|c|}
\hline 1900 & $25-50$ & $50-75$ & $75-100$ & $100-125$ \\
\hline $25-50 \ldots \ldots$ & $\begin{array}{l}\text { Soria Huesca } \\
\text { Cuenca Teruel } \\
\text { Albacete } \\
\text { Cáceres } \\
\text { Guadajara }\end{array}$ & Ciudad Real & & \\
\hline $50-75$ & $\begin{array}{l}\text { Burgos } \\
\text { Lérida }\end{array}$ & $\begin{array}{l}\text { Segovia Toledo } \\
\text { Huelva Zamora } \\
\text { Badajoz Avila } \\
\text { Zaragoza León } \\
\text { Palencia } \\
\text { Salamanca }\end{array}$ & & \\
\hline $75-100 . \ldots$ & & $\begin{array}{l}\text { Alava } \\
\text { Navarra }\end{array}$ & Valladolid & $\begin{array}{l}\text { Zórdoba } \\
\text { Jaén }\end{array}$ \\
\hline
\end{tabular}

\begin{tabular}{|c|c|c|c|c|}
\hline 1975 & $25-50$ & $50-75$ & $75-100$ & $100-125$ \\
\hline Menos de 25 & $\begin{array}{l}\text { Cuenca } \\
\text { Guadalajara } \\
\text { Huesca } \\
\text { Soria } \\
\text { Teruel }\end{array}$ & & & \\
\hline $25-50 \ldots$ & $\begin{array}{l}\text { Albacete } \\
\text { Burgos } \\
\text { Cáceres } \\
\text { Lérida }\end{array}$ & 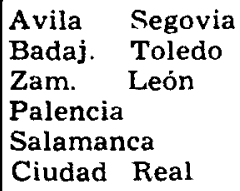 & & \\
\hline $50-75 \ldots$ & & $\begin{array}{l}\text { Huelva } \\
\text { Zaragoza } \\
\text { Navarra }\end{array}$ & & $\begin{array}{l}\text { Córdoba } \\
\text { Jaén }\end{array}$ \\
\hline $75-100$. & & & Valladolid & \\
\hline $100-125 \quad \ldots \quad \ldots$ & & Alava & & \\
\hline
\end{tabular}




\section{CUADRO 5}

Provincias que en 1900 tenian densidad superior a la media nacional.

Evolución de densidades

\begin{tabular}{ll|l|l|l|l|l|l|l}
\hline & & & & & \\
\hline
\end{tabular}


CUADRO 5 (Continuación)

\begin{tabular}{ll|l|l|l|l|l|l|l}
\hline & & & & & & \\
\hline
\end{tabular}


El cuadro 5 hace referencia a las provincias de densidad superior a la nacional y se ha construido de la misma forma que el cuadro anterior.

De las provincias periféricas sólo Huelva tiene densidad inferior a la nacional, en 1900. Sin embargo, la dinámica de los dos períodos señalados es aquí bien diferente a la que aparecía en el cuadro 4 . Aquí, siete provincias periféricas pierden población, en términos relativos, entre 1900 y 1950, y sólo cuatro en el último período. Ello quiere decir que el proceso de concentración de la población en determinadas áreas periféricas no se genera a partir de 1950, sino que ya aparecía desde principios de siglo. Es más, el proceso de concentración es más polarizado en los primeros cincuenta años. A partir del Plan de Estabilización, los polos de concentración se amplían a las provincias limítrofes, como pasa en Gerona, Tarragona, etc., y de ahí que el número de provincias que pierden población, en términos relativos, sea menor.

Todo lo anterior lleva a la conclusión de que hablar de provincias interiores y exteriores en España es hablar de dos mundos distintos demográficamente, tanto por el volumen de población como por su ritmo de crecimiento.

Trasladado todo ello a nuestro análisis regional queda bien patente el abismo que separa, demográficamente, a unas regiones de otras, tal como se señaló páginas atrás y se ha ido perfilando ahora con esta breve incursión en el análisis provincial.

\section{Estructura de edades y proceso de reproducción de la población}

Los datos anteriores ofrecen una situación bastante sombría, demográficamente, para muchas regiones. Sin embargo, estos datos son incompletos, ya que no reflejan la estructura de la población ni el dinamismo del proceso reproductor. Un estudio, en esta dirección, ayuda a completar la visión anterior y permite un análisis más cualitativo de las diferencias entre regiones a que se viene aludiendo.

La estructura de edades se ha modificado sensiblemente en los quince años que van de 1960 a 1975 . Esta modificación ha ido en la dirección de un continuo envejecimiento de la población de las provincias agrícolas, es decir, las menos desarrolladas.

Una primera aproximación al problema se obtiene a través de un análisis de correlaciones ${ }^{17}$ entre saldos migratorios y porcentaje de población de menos de quince años y más de sesenta y cuatro años. Los resultados son éstos:

"Se han hecho tres matrices de correlaciones, una para cada año, utilizando. para cada variable, los datos de las 50 provincias. 
Correlación entre saldos migratorios y población de menos de 15 años y más de 64

\begin{tabular}{|c|c|c|c|}
\hline \multirow{2}{*}{\multicolumn{2}{|c|}{ Años }} & \multirow[b]{2}{*}{$\begin{array}{c}\text { Menos } \\
\text { de } 15 \text { años }\end{array}$} & \multirow[b]{2}{*}{$\begin{array}{c}\text { Más } \\
\text { de } 64 \text { ajios }\end{array}$} \\
\hline & & & \\
\hline $1960 \ldots$ & $\begin{array}{lllll}\ldots & \ldots & \ldots & \ldots & \ldots\end{array}$ & -.33 & -.08 \\
\hline $1970 \ldots$ & $\begin{array}{lllll}\ldots & \ldots & \ldots & \ldots & \ldots\end{array}$ & .01 & -.42 \\
\hline $1975 \ldots$ & $\begin{array}{lllll}\ldots & \ldots & \ldots & \ldots & \ldots\end{array}$ & .39 & -.59 \\
\hline
\end{tabular}

Las provincias con saldos migratorios positivos son las más desarrolladas, las que constituyen las regiones de máximo dinamismo, y las de saldos negativos las menos desarrolladas, las de mayor porcentaje de población agrícola.

La correlación entre saldos migratorios y población de menos de 15 años es muy baja, pero, sin embargo, muestra un cambio de tendencia altamente significativo para nuestro propósito. En 1960 las provincias más desarrolladas son las que tienen menor porcentaje de población en esas edades, pero, quince años más tarde, la correlación cambia de signo, siendo en 1975 las provincias más desarrolladas las que tienen mayor población menor de 15 años. En 1960 no aparece correlación entre saldos migratorios y población mayor de 64 años, pero esta correlación se va haciendo presente con el paso del tiempo, haciéndose notoria en 1975.

La conclusión del análisis de ambas correlaciones es evidente. A lo largo de quince años ha ido aumentando la población joven en las provincias desarrolladas y ha ido aumentando la población de mayor edad en las provincias deprimidas. Se ha dado, por tanto, una modificación sustancial en la estructura de edades de unas y otras provincias en el sentido de un mayor envejecimiento de las provincias regresivas, que constituyen las regiones interiores que aparecen en los últimos lugares del cuadro 1.

A esta misma conclusión se llega por otro camino y para ello se ha confeccionado el cuadro 6 . Como se observa en el mismo el porcentaje de población de menos de 15 años ha aumentado en las seis primeras regiones, de 1960 a 1975, y ha disminuido en las restantes, salvo en el caso de Murcia. En cuanto a la población de 65 años y más, ha aumentado en todos los casos, de 1960 a 1975 , pero este aumento es muy distinto de unas a otras regiones. Mientras que en 1960 no aparece una diferencia importante de porcentajes entre las regiones desarrolladas y no desarrolladas, sin embargo, en 1975 aparece esta diferencia de forma notoria. La diferencia de porcentaje entre 1960 y 1975 gira en torno a un punto en las regiones desarrolladas y en torno a cuatro o cinco en el caso de las últimas regiones del cuadro, es decir, las menos desarrolladas. 
No parece necesario insistir en este cambio de tendencia, ya que los datos lo muestran de una forma diáfana. La población de las regiones menos desarrolladas ha envejecido considerablemente en los últimos quince años y ha envejecido también en comparación a la de las regiones desarrolladas.

CUADRO 6

Estructura de edades

\begin{tabular}{|c|c|c|c|c|c|c|}
\hline \multirow{2}{*}{ Regiones } & \multicolumn{3}{|c|}{ De 0 a 14 años } & \multicolumn{3}{|c|}{ De 65 y más años } \\
\hline & 1960 & 1970 & 1795 & 1960 & 1970 & 1975 \\
\hline $\begin{array}{lllllll}\text { Madrid } & \ldots & \ldots & \ldots & \ldots & \ldots & \ldots\end{array}$ & 25,2 & 28,0 & 28,2 & 6,8 & 7,9 & 8,3 \\
\hline País Vasco $\ldots \ldots \ldots \ldots \ldots$ & 27,7 & 28,9 & 27,9 & 7,1 & 7,7 & 8,2 \\
\hline $\begin{array}{llllll}\text { Cataluña } & \ldots & \ldots & \ldots & \ldots & \ldots\end{array}$ & 23,2 & 25,6 & 25,6 & 9,5 & 9,8 & 10,2 \\
\hline $\begin{array}{llllll}\text { Canarias } & \ldots & \ldots & \ldots & \ldots & \ldots\end{array}$ & 32,8 & 33,2 & 33,2 & 6,3 & 6,8 & 7,4 \\
\hline $\begin{array}{lllllll}\text { Valencia } & \ldots & \ldots & \ldots & \ldots & \ldots\end{array}$ & 24,6 & 26,8 & 27,0 & 9,6 & 10,2 & 10,8 \\
\hline $\begin{array}{lllllll}\text { Baleares } & \ldots & \ldots & \ldots & \ldots & \ldots & \ldots\end{array}$ & 21,4 & 24,3 & 25,2 & 11,6 & 11,8 & 12,2 \\
\hline $\begin{array}{lllllll}\text { Navarra } & \ldots & \ldots & \ldots & \ldots & \ldots & \ldots\end{array}$ & 27,5 & 25,8 & 26,0 & 9,5 & 10,5 & 10,8 \\
\hline $\begin{array}{lllllll}\text { Asturias } & . & \ldots & \ldots & \ldots & \ldots & \ldots\end{array}$ & 27,1 & 25,7 & 23,3 & 7,8 & 9,7 & 11,3 \\
\hline $\begin{array}{llllll}\text { Santander } & \ldots & \ldots & \ldots & \ldots & \ldots\end{array}$ & 28,5 & 28,0 & 25,2 & 8,1 & 10,0 & 11,1 \\
\hline $\begin{array}{lllllll}\text { Murcia } & \ldots & \ldots & \ldots & \ldots & \ldots & \ldots\end{array}$ & 29,2 & 31,1 & 30,2 & 8,0 & 9,5 & 10,1 \\
\hline $\begin{array}{lllllll}\text { Galicia } & \ldots & \ldots & \ldots & \ldots & \ldots & \ldots\end{array}$ & 26,7 & 24,7 & 23,7 & 8,9 & 11,3 & 12,3 \\
\hline $\begin{array}{llllll}\text { Andalucia } & \ldots & \ldots & \ldots & \ldots & \ldots\end{array}$ & 30,7 & 31,6 & 30,4 & 7,2 & 8,6 & 9,4 \\
\hline $\begin{array}{lllllll}\text { Logroño } & \ldots & \ldots & \ldots & \ldots & \ldots & \ldots\end{array}$ & 25,8 & 23,5 & 23,7 & 8,4 & 11,5 & 12,3 \\
\hline $\begin{array}{lllllll}\text { Aragón } & \ldots & \ldots & \ldots & \ldots & \ldots & \ldots\end{array}$ & 23,8 & 23,5 & 22,8 & 9,8 & 12,1 & 13,4 \\
\hline $\begin{array}{llllll}\text { Castilla-León } & \ldots & \ldots & \ldots & \ldots\end{array}$ & 29,1 & 26,5 & 24,2 & 8,3 & 10,8 & 12,7 \\
\hline $\begin{array}{llllll}\text { La Mancha } & . . & \ldots & \ldots & \ldots & \ldots\end{array}$ & 28,6 & 27,7 & 26,2 & 8,4 & 11,5 & 12,8 \\
\hline $\begin{array}{lllll}\text { Extremadura } & \ldots & \ldots & \ldots & \ldots\end{array}$ & 29,7 & 28,5 & 26,6 & 7,6 & 10,7 & 12,5 \\
\hline TOTALES $\ldots \ldots \ldots$ & 27,3 & 27,8 & 27,1 & 8,2 & 9,7 & 10,5 \\
\hline
\end{tabular}

Fuente: Explotación de datos censales y del Padrón de 1975.

El proceso de reproducción de la población, medido por el crecimiento vegetativo, muestra la misma tendencia que en el caso anterior, al cual está lógicamente ligado. Aparte de otros muchos factores de orden cultural y económico, el proceso de reproducción está muy ligado a la estructura de edades, ya que si desciende, en términos relativos, la población en edad de reproducir, desciende por el mismo hecho, no modificándose los demás factores que influyen en el comportamiento reproductor, la natalidad. 
El cambio de tendencia se puede observar a través del análisis de correlaciones, como en el caso anterior. Ahora se ha tomado como punto de referencia el porcentaje de activos agrícolas, como indicador de desarrollo. La correlación entre esta variable y la TBN, la TBM y el Crecimiento Vegetativo ha ido evolucionando de la siguiente forma:

\begin{tabular}{|c|c|c|c|c|}
\hline \multirow{2}{*}{\multicolumn{2}{|c|}{$A \bar{n} \circ \mathrm{s}$}} & \multicolumn{3}{|c|}{$\begin{array}{c}\text { Correlación entre \% agrícolas } \\
y \text { TBN, TBM y CV. }\end{array}$} \\
\hline & & $T B N$ & $T B M$ & $C V$ \\
\hline $\begin{array}{lll}1960 & \ldots & . . \\
1970 & \ldots & . . \\
1975 & \ldots & . .\end{array}$ & $\begin{array}{ccccccc}\ldots & \ldots & \ldots & \ldots & \ldots & \ldots & \ldots \\
\ldots & \ldots & \ldots & \ldots & \ldots & \ldots & \ldots \\
\ldots & \ldots & \ldots & \ldots & \ldots & \ldots & \ldots\end{array}$ & $\begin{array}{l}-.08 \\
-.53 \\
-.55\end{array}$ & $\begin{array}{l}.10 \\
.46 \\
.68\end{array}$ & $\begin{array}{l}-.11 \\
-.23 \\
-.60\end{array}$ \\
\hline
\end{tabular}

En los tres casos se observa cómo en el transcurso de quince años aparece una correlación clara, pero negativa, con la TBN, una correlación positiva con la TBM y una correlación negativa con el CV. Es decir, en 1975, las provincias agrícolas son las de menor TBN, las de mayor TBM y, consecuentemente, las de menor CV. Esta asociación no aparecía quince años atrás. Si se tratara de un análisis puramente demográfico habría que seguir desarrollando este apartado, pero para nuestro intento de analizar los recursos humanos de cada región y su dinámica, parece suficiente lo aquí expuesto. Baste añadir que el crecimiento vegetativo ha aumentado en algunas de las regiones más desarrolladas, mientras que en algunas regiones menos desarrolladas sigue una tendencia claramente regresiva, aproximándose paulatinamente al crecimiento 0 , tal como ocurre en Aragón, con la excepción de Zaragoza, en Castilla-León, con la excepción de Valladolid, en la Mancha y Extremadura.

La conclusión última a que se llega al final de este análisis es clara. Determinadas regiones han ido ganando continuamente población a costa del resto, la relación hombre/espacio es muy fuerte en las regiones desarrolladas y muy baja y en regresión en las estancadas económicamente, la población de unas regiones rejuvenece, en términos relativos, mientras que la de otras envejece, el proceso reproductor sigue siendo dinámico o adquiere nuevo dinamismo en las regiones tradicionalmente desarrolladas y languidece en las regiones agrícolas y despobladas. Este es el panorama de las regiones españolas, desde el punto de vista demográfico, con el que nos encontramos a final de 1975, años a que hacen referencia los últimos datos analizados.

\section{PROCESO DEMOGRAFICO, PROCESO ECONOMICO}

En las páginas anteriores se ha ido dejando constancia de que existe relación entre variables económicas y demográficas, de tal forma que deter- 
minado comportamiento de las variables económicas se corresponde con determinado comportamiento de las variables demográficas. Dado que, en el caso de España, el proceso demográfico y el económico han ido paralelos y la explosión de ambos, en nuestros tiempos, se produce en la misma época, parece del máximo interés apuntar alguna de las relaciones entre economía y demografía, partiendo del proceso de redistribución de la población, analizado en la primera parte de este trabajo.

\section{Análisis de correlaciones}

En un trabajo anterior ${ }^{18}$ había realizado una matriz de correlaciones, referida a 1970, en la que, partiendo de datos de las cincuenta provincias, analizaba fundamentalmente variables demográficas. Ahora se ha repetido dicha matriz, ampliando considerablemente el número de variables e incluyendo muchas más económicas (véase cuadro 7 ).

\section{CUADRO 7}

Matriz de correlaciones año 1970

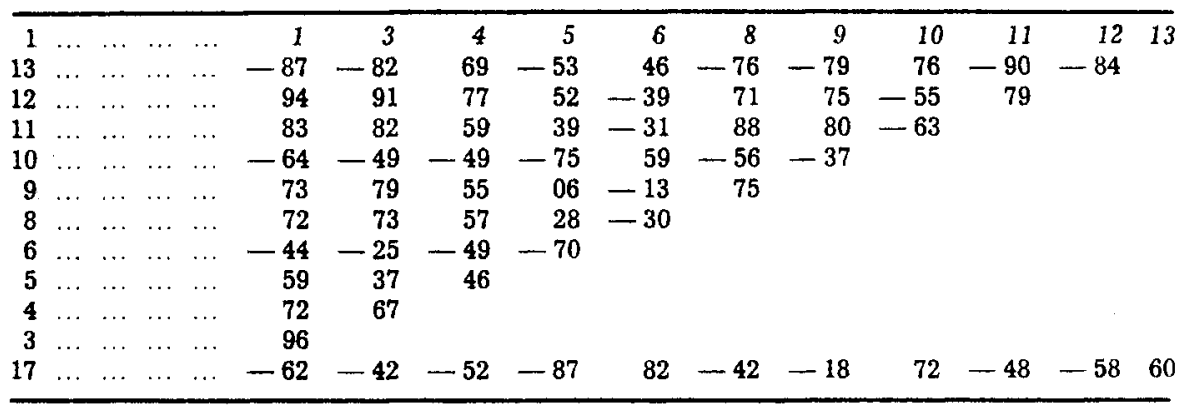

Definición de las variables

1. Crecimiento poblacional intercensal.

3. Saldo migratorio intercensal.

4. Densidades provinciales.

5. TBN (año de referencia).

6. TBM (año de referencia).

8. Producción manufacturera en relación a la producción provincial (año de referencia.

9. RPC (año de referencia).

10. Población activa no asalariada (empresarios $\sin$ asalariados y trabajadores independientes) en relación a la población activa (año de referencia).

11. Obreros calificados en relación a la población activa (año de referencia).

12. Evolución población activa en índices (crecimiento intercensal).

13. Población activa en agricultura (año de referencia).

17. Población de más de 65 años (año de referencia). 1978.

Jacinto Rodrfguez Osuna: Población y desarrollo en España, Cupsa, Madrid. 
Tomando como referencia el crecimiento intercensal, que es el que más ha variado de unas a otras provincias, se puede dibujar un perfil, en que se destacan los rasgos demográficos y económicos más importantes de las provincias de máximo crecimiento intercensal positivo y más desarrolladas, y los de las provincias de máximo crecimiento intercesal negativo, las menos desarrolladas. El resultado de dicho perfil, que no es más que una lectura parcial de la matriz, podría ser el que aparece en el cuadro 8 .

\section{CUADRO 8}

Perfil de las provincias desarrolladas y de las regresivas

\begin{tabular}{|c|c|c|}
\hline \multirow{2}{*}{ Variables } & \multicolumn{2}{|c|}{ Provincias } \\
\hline & Desarrolladas & Regresivas \\
\hline \multicolumn{3}{|l|}{ Demográficas: } \\
\hline 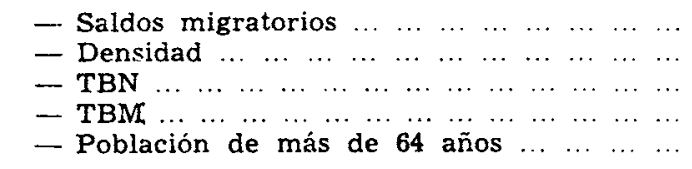 & $\begin{array}{l}\text { Positivos } \\
\text { Alta } \\
\text { Alta } \\
\text { Baja } \\
\text { Or bajo }\end{array}$ & $\begin{array}{l}\text { Negativos } \\
\text { Baja } \\
\text { Baja } \\
\text { Alta } \\
\% \text { alto }\end{array}$ \\
\hline \multicolumn{3}{|l|}{ Económicas: } \\
\hline 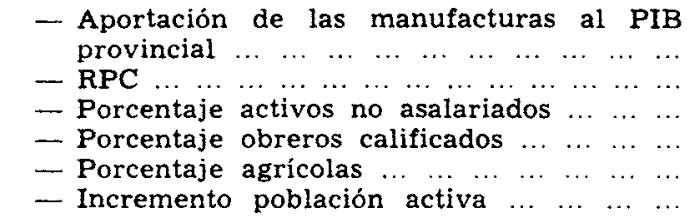 & $\begin{array}{l}\text { Alta } \\
\text { Alta } \\
\text { Bajo } \\
\text { Alto } \\
\text { Bajo } \\
\text { Positivo }\end{array}$ & $\begin{array}{l}\text { Baja } \\
\text { Baja } \\
\text { Alto } \\
\text { Bajo } \\
\text { Alto } \\
\text { Negativo }\end{array}$ \\
\hline
\end{tabular}

En dicho cuadro no se cuantifican los valores de cada variable, pero sí aparece su comportamiento en unas y otras provincias en términos comparativos. Si se continuara el trabajo por este camino se podría señalar cuándo va apareciendo el comportamiento diferencial de cada variable y qué valores va adquiriendo su distanciamiento. Aunque ello sería interesante, lo que ahora hay que resaltar, sobre todo, es que existe una correspondencia entre proceso económico y demográfico en el caso de las provincias y regiones españolas.

\section{El desarrollo económico desigual, causa original del proceso}

En la base de la redistribución espacial de la población española están las migraciones interiores y en el origen de las migraciones el desarrollo 
económico desigual de las provincias. Una vez iniciado el proceso, las migraciones van a ir alimentando el sistema productivo de las regiones desarrolladas, suministrando el trabajo necesario para el elevado ritmo de crecimiento experimentado. El proceso económico distinto, de unas y otras regiones, es la causa inicial del distanciamiento demográfico vía migraciones ${ }^{19}$.

Sobre las migraciones y sus causas se ha escrito mucho, siendo imposible recoger aquí la dirección de las distintas investigaciones. Sin embargo, en el caso de España, podría centrarse el análisis en estas direcciones: las migraciones interiores obedecen a un:

- Proyecto de mejora de rentas.

- Proyecto de mejora de condiciones de trabajo.

- Proyecto de mejora de condiciones de vida.

Las diferencias de RPC en 1955 es muy elevada de una a otra provincia, de tal forma que en este año la RPC de Vizcaya es 4,18 veces la de Orense; veinte años más tarde, la distancia de RPC entre las dos provincias extremas se habrá reducido considerablemente, siendo la RPC de Vizcaya 2,36 veces la de Cáceres, provincia de menor renta en este año. ¿Qué ha ocurrido?

Lo que ha ocurrido es que la población se ha trasladado a los lugares de mayor generación de rentas y, como consecuencia, se ha mejorado la renta de las regiones de origen. En el fondo del problema está la productividad desigual, que depende de la desigual estructura del sistema productivo. En determinadas regiones, las industriales, la productividad es elevada, y en otras, la mayor parte de las agrícolas, muy baja. Ante la diferencia de rentas, motivada por la diferencia de productividades, se originan las migraciones, que sólo se atenúan cuando se cierran los mercados de trabajo o cuando se atenúan las desigualdades.

Las migraciones inciden en la relación hombre/espacio y debido a ellas se ha dado la polarización de la población en determinadas áreas, lo que obedecería a la búsqueda de un equilibrio entre población y recursos, $\mathrm{o}$, dicho de otra forma, a la búsqueda de un óptimo de población hipotético para cada área.

Aunque las investigaciones sobre el óptimo de población no han avan zado empíricamente, sin embargo, se puede sostener la hipótesis de que existiendo movilidad de la mano de obra se da un cierto ajuste entre población y recursos en explotación, lo que motiva el traslado de la población a aquellos lugares en que sus posibilidades de trabajo y sus rentas se hagan mayores. Si esto es así, en el momento en que en una determinada área exista un exceso de población en relación a los recursos generados por la economía,

"Las migraciones pueden obedecer a distintos proyectos. Las que aquí se analizan obedecen fundamentalmente a un proyecto de mejora económica. 
esa población tenderá a emigrar a otras áreas en que pueda encontrar mayores posibilidades de trabajo y rentas. En este sentido, la medida de la situación óptima no va a venir determinada por la densidad de población, sino por la relación recursos-población.

Parece ser que este esquema elemental ha venido desarrollándose al menos a lo largo de este siglo en las provincias españolas y que los desplazamientos de población han respondido a estos condicionamientos.

La España interior y la España periférica, con sus distintas situaciones demográficas tan acusadas, no serían más que una consecuencia de esa búsqueda de situaciones óptimas en que el factor determinante sería el económico.

La mejora de condiciones de trabajo y la mejora de condiciones de vida están también en el origen de las migraciones. Su incidencia es menor en el caso de España, aunque depende de diferentes factores, entre los que interviene de forma importante la cualificación del emigrante, su posición en la estratificación ocupacional. Sobre el tema se han hecho diferentes investigaciones, pero, para nuestro propósito de relacionar economía y demografía, el tema tiene menos interés en este trabajo.

\section{Las migraciones y los cambios en la estructura de la población}

Las migraciones inciden en la estructura de edades, en el proceso reproductor y en la futura dinámica de las migraciones.

Los cambios en la estructura de edades y en el proceso reproductor han sido analizados en la primera parte de este trabajo, aunque sin insistir en su relación directa con las migraciones. Esta se produce a través de una serie de interrelaciones que quedan reflejadas en el esquema de la página siguiente. Los datos que apoyan la secuencia que aparece en este esquema han quedado reflejados páginas atrás.

La incidencia de las migraciones en la futura evolución de las mismas, tratándose de migraciones de origen económico, también aparece en el esquema. En un principio, las migraciones generan nuevas migraciones por la sencilla razón de que en los lugares de destino contribuyen a dinamizar la economía con la consecuente continua creación de nuevos puestos de trabajo; en los lugares de origen producen un efecto demostración y una ruptura de la inercia que da lugar a una emigración continuada.

Los procesos migratorios, sin embargo, tienen un límite que viene señalado en el esquema que se ha realizado a partir de la experiencia española. En los lugares de destino aumenta, en términos relativos, el crecimiento vegetativo, y, en consecuencia, aumenta la población activa, que va a alimentar el propio proceso productivo; por otra parte, la diferencia de productividad desciende $\mathrm{y}$, por tanto, una parte de los atractivos de la migración. Si a esto se une el descenso de la demanda de trabajo, como consecuencia 


\section{Efectos de las migraciones en distintas variables demográficas}

Provincias no desarrolladas

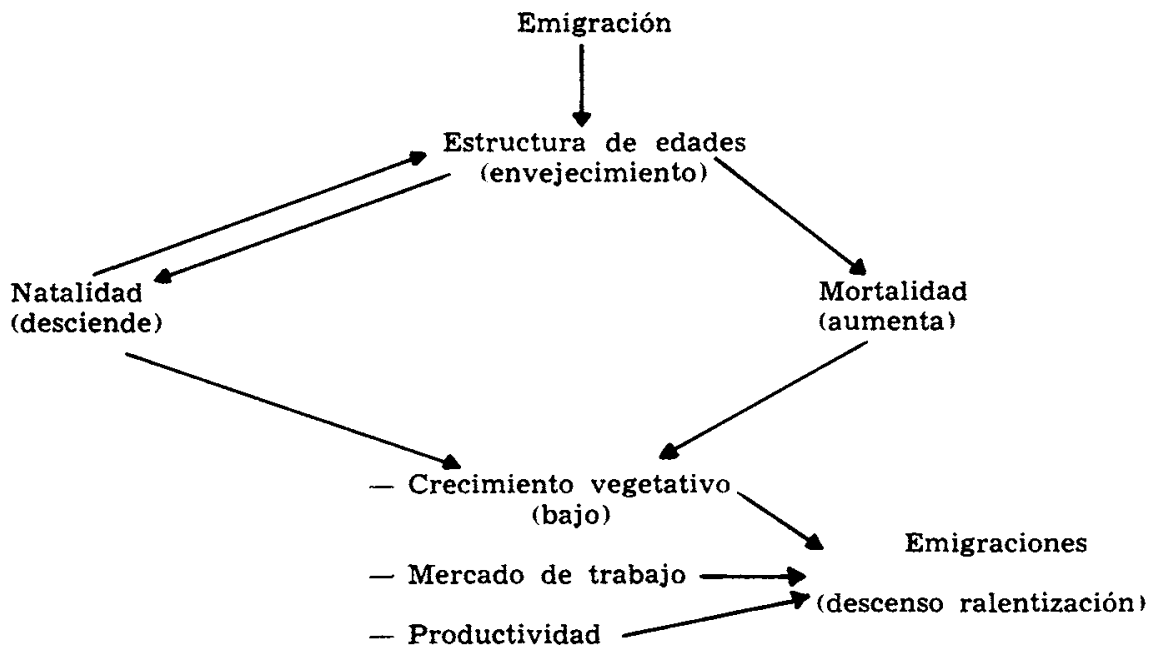

Provincias desarrolladas

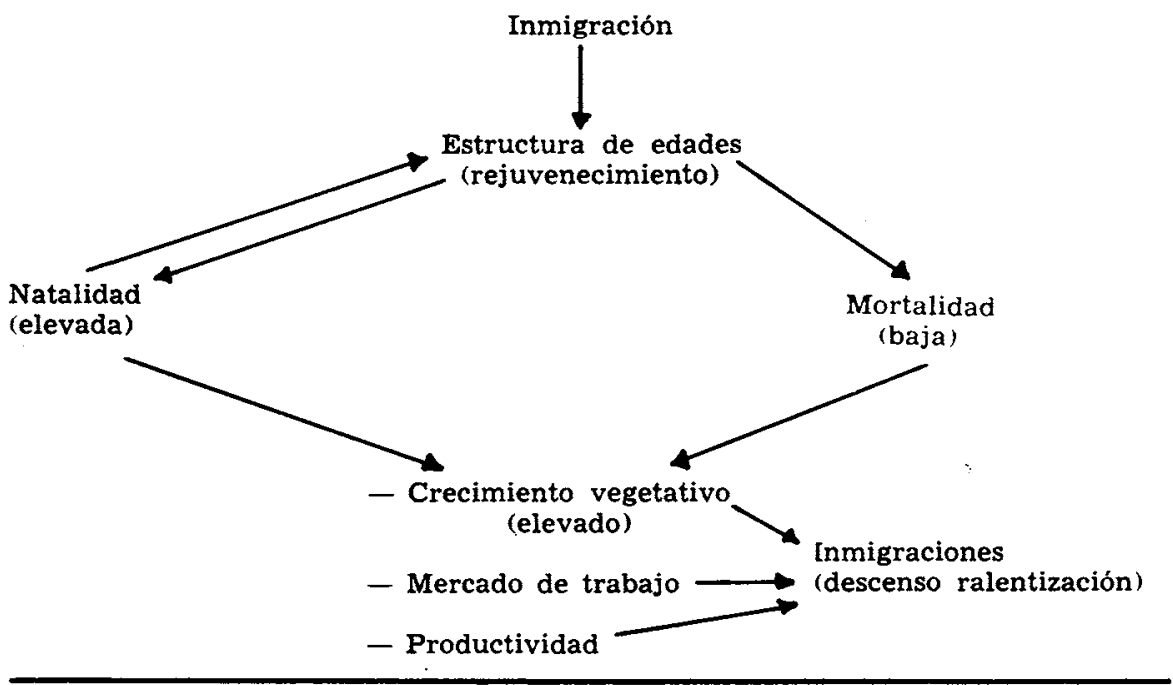


de la saturación industrial, la aparición de deseconomías externas y quizá la aparición de la crisis, como en la actualidad, se explica fácilmente el descenso de las migraciones.

En los lugares de origen se dan las situaciones contrarias, el crecimiento vegetativo se aproxima a cero y, por tanto, no se generan nuevos excedentes de población, la productividad y las rentas aumentan comparativamente, los incentivos de la emigración disminuyen. 